\title{
Synthesis of chiral inducers having double stereogenic centers for electrochemical polymerization in cholesteric liquid crystal medium
}

\author{
Tomokazu Iseki, ${ }^{1}$ Kohsuke Kawabata, ${ }^{1}$ Shigeki Nimori, ${ }^{2}$ Hiromasa Goto ${ }^{1} *$
}

${ }^{1}$ Division of Materials Science, Faculty of Pure and Applied Sciences, University of Tsukuba, University of Tsukuba, Tsukuba, Ibaraki 305-8573, Japan

${ }^{2}$ Tsukuba Magnet Laboratory, National Institute for Materials Science, Tsukuba, Ibaraki 305-0003, Japan

Correspondence to H. Goto, e-mail: gotoh@ims.tsukuba.ac.jp, fax: +81-29-853-4490

\begin{abstract}
An enantiomeric pair of chiral inducers with double chiral carbons was synthesized by a simple three-step reaction strategy. The process affords the chiral inducers with good yield, high enantiomeric excess, and good compatibility with nematic liquid crystal. Addition of a small amount of the chiral inducers thus synthesized in this study produced cholesteric architecture. Electrochemical polymerization in the asymmetric liquid crystal environment affords chiroptically active polymers as atropisomers. The electrochemical polymerization under magnetic field of $4 \mathrm{~T}$ affords polymer films with linear dichroism. The polymers exhibit a significant color change from dark-green in the oxidized state to light-green in the reduced state. The redox process of the polymer films in an electrolyte provides a reversible absorption, circular dichroism, and linear dichroism spectral changes.
\end{abstract}

Keywords; Chiral inducer, Liquid crystal, Optical activity

\section{Introduction}

Conjugated polymers have been extensively studied since the discovery of electrical conductivity in doped polyacetylene [1]. Electrochemically conductive polymers based on polythiophene and polypyrrole led to their use in a wide range of organic electronic devices including ion gates, and electrochemical diodes [2], as well as electrochromic devices [3], electro-luminescent devices [4], electrodes and batteries [5], and sensors [6,7]. Electrochemical polymerization is one of the most useful methods for preparing conjugated polymers because this method provides a polymer film easily and conveniently on an electrode surface [8]. The polymer displays redox properties and electrochromism [9]. 
The synthesis of optically active polymers has generated great interest in macromolecular science due to a wide variety of potential applications based on the chiral structure. Optically active polymers have been obtained by several methods, including the polymerization of optically active monomers [10], asymmetric selective polymerization [11], and non-covalent interaction between optically inactive polymers and chiral molecules [12]. Macromolecular optical activity originates from the helical structure of the polymer, is an attractive biomimetic technology and holds promise for prospective applications, such as asymmetric separation and recognition properties [13].

In our previous studies, electrochemical polymerization in cholesteric liquid crystal (CLC) phase afforded helically ordered conjugated polymer films [14]. The polymerization proceeds epitaxially on the electrode surface, which enables growth of the polymer film aligned with the liquid crystal (LC) molecular order. The individual molecules of CLC aggregate in a three-dimensional (3-D) one-handed helical structure, resulting in structural chirality. On the other hand, the electrochemical polymerization under magnetic field affords polymer films with linear polarized electrochromism [15]. The polymer transcribes the molecular arrangement of the LC molecules by an applied magnetic field.

The addition of chiral molecules to nematic LC can induce the CLC architecture. Cholesterol esters have been widely used as additives for induction of helical structure in the nematic LC. However, the use of cholesterol derivatives is limited for the construction of a pair of mirror image helical architecture, because of synthetic difficulty for enantiomeric pairs of cholesterol derivatives. Therefore, the synthesis of a pair of mirror image chiral inducers is important, which are easily accessible, chemically more robust, and widely compatible with any type of nematic LC.

In this research, an enantiomeric pair of chiral inducers with two chiral carbons was synthesized using a simple three-step reaction. Mitsunobu reactions were employed to prepare the chiral inducers. This reaction is found to be particularly effective at inverting the configuration of chiral secondary alcohols [16]. Consequently, the chiral inducers were obtained with high enantiomeric excess (ee). Helical twisting power (HTP) of the chiral inducers was estimated by the Cano-wedge cell method [17]. The electrochemical polymerization of 1,4-di(2-thienyl)benzene (TBT) was performed in CLC electrolyte under magnetic field. The CLC electrolyte solution was induced using the chiral inducers. Poly(TBT) synthesized under $0 \mathrm{~T}$ displayed a fingerprint texture similar to that of the CLC electrolyte solution. Poly(TBT) synthesized under $4 \mathrm{~T}$ exhibited a stripe texture.

Addition of a small amount of the chiral inducers thus synthesized in this study 
produced chiral LC from achiral nematic of LC solvent. Polymerization in the asymmetric LC environment afforded chiroptically active polymers as atropisomers. In other words, induction by the chiral inducers via environmental change resulted in formation of helical structure of the resultant polymer. The polymerization can be referred to as structure-organized polymerization (SOP). The SOP in this study is derived from the inducers with double chiral centers (double chiral inducers). We report synthesis of the double chiral inducers and electrochemical polymerization by using these compounds for asymmetric environment.

\section{Experimental}

\subsection{Instrumentation and materials}

The chemical structure of all intermediates and final products were confirmed by the ${ }^{1} \mathrm{H}$ NMR and ${ }^{13} \mathrm{C}$ NMR spectra (ECS 400 spectrometer using $\mathrm{CDCl}_{3}$ as the deuterated solvent, and tetramethylsilane (TMS) as the internal standard), infrared spectroscopy (Jasco FT-IR 500 spectrometer using $\mathrm{KBr}$ method) and elementary analysis (Perkin-Elmer 2400 CHN Elemental Analyzer). Enantiomeric excess was determined by HPLC analysis with a chiral column (CHIRALPAK ${ }^{\circledR}$ IC). Optical rotations of compounds were measured with Jasco P-1010 Polarimeter using tetrahydrofuran (THF) as the solvent. Helical twisting power $(\beta)$ of the CLC was obtained by using the Cano-wedge cell (EHC, KCRK-03, $\tan \theta=0.0083$ ). Electrochemical measurements of polymers were performed using an electrochemical analyzer PGSTAT 12 (AUTOLAB, the Netherlands). Optical texture observations were carried out using a Nikon ECLIPS LV 100 high resolution polarizing microscope with a Nikon LU Plan Fluor lens and a Nikon CFIUW lens. Magnetic orientation was carried out with a drum type cryogen-free superconducting magnet (Japan Magnet Technology, JMT). Circular dichroism (CD), linear dichroism (LD) (Jasco, J-720 spectrometer) and ultraviolet visible (UV-vis) spectra (Jasco, U-3500 spectrophotometer) were recorded. The spin densities of the monomer in the radical cationic state was calculated by density functional theory (DFT) method at the B3LYP/631G*.

All reagents were used after further purification by standard methods. Methyl 4-carboxyphenyl carbonate (1) [18], TBT [19] and 4-cyano-4'-octyloxybiphenyl (8OCB) [15] were prepared as previously reported. The compounds 2-octanol, diethyl azodicarboxylate, triphenylphosphine, ammonia, cholesteryl oleyl carbonate and 
tetrabutylammonium perchlorate (TBAP) were purchased from Tokyo Kasei Kogyo Co., Ltd. The 4-cyano-4'-hexylbiphenyl (6CB) was purchased from Merck. Hexane, acetonitrile, chloroform, THF and ethanol (EtOH) were purchased from Nacalai Tesque Co., Ltd. Silica gel $60 \mathrm{~N}$ was purchased from Kanto Chemical Co., INC.

\section{Synthesis}

\subsubsection{Syntheses of Chiral inducers}

\subsubsection{1. (S)-4-(1-methyl carbonic acid)-benzoic acid 1-methyl-heptyl ester (S)-2}

$(R)$-(-)-2-octanol (1.33 g, $10.2 \mathrm{mmol})$ and diethyl azodicarboxylate (5.67 g, $28.0 \mathrm{mmol})$ were slowly added dropwise to a stirred mixture of triphenylphosphine $(3.21 \mathrm{~g}, 12.2$ $\mathrm{mmol}$ ) and methyl 4-carboxyphenyl carbonate (1) (2.01 g, $10.2 \mathrm{mmol})$ in dry THF (20 $\mathrm{mL}$ ) under argon at $0{ }^{\circ} \mathrm{C}$. The reaction mixture was warmed up to room temperature and stirred for $24 \mathrm{~h}$. After the completion of the reaction was checked by TLC, the resulting solution was extracted with chloroform. After drying over $\mathrm{MgSO}_{4}$ and filtration, chloroform was removed using a rotary evaporator. The crude product was purified by silica gel column with chloroform to give a colorless oil $(2.67 \mathrm{~g}, 8.7 \mathrm{mmol}$, yield $=$ $87 \%$ ), which was dried in vacuo. ${ }^{1} \mathrm{H}$ NMR (400 MHz, $\delta$ from TMS (ppm), $\mathrm{CDCl}_{3}$ ): $\delta$ $0.80(\mathrm{t}, 3 \mathrm{H}), 1.25(\mathrm{~m}, 11 \mathrm{H}), 1.59(\mathrm{~m}, 2 \mathrm{H}), 3.85(\mathrm{~s}, 3 \mathrm{H}), 5.07$ (sextet, 1H), $7.17(\mathrm{~d}, 2 \mathrm{H})$, $8.00(\mathrm{~d}, 2 \mathrm{H}) .{ }^{13} \mathrm{C}$ NMR (400 MHz, $\delta$ from TMS (ppm), $\left.\mathrm{CDCl}_{3}\right): \delta 165.2,154.3,153.6$, $131.1,128.7,120.8,72.0,55.6,36.0,31.7,29.1,25.4,22.6,20.1,14.1 .[\alpha]_{589}{ }^{\mathrm{RT}}=-3.21^{\mathrm{o}}$ $(0.0100 \mathrm{~g} / \mathrm{mL}$; THF) for $96 \%$ ee.

\subsubsection{2. (R)-4-(1-methyl carbonic acid)-benzoic acid 1-methyl-heptyl ester $(\boldsymbol{R})$-2}

This compound was prepared using the same method that was described for $(\boldsymbol{S}) \mathbf{2}$. Quantities used: triphenylphosphine (3.19 g, $12.2 \mathrm{mmol}),(S)$-(+)-2-octanol (1.60 g, 12.3 mmol), methyl 4-carboxyphenyl carbonate (1) (2.01 g, $10.2 \mathrm{mmol})$, diethyl azodicarboxylate $(5.68 \mathrm{~g}, 28.1 \mathrm{mmol})$ and dry THF $(20 \mathrm{~mL})$. Quantities yield: colorless oil (2.78 g, $9.0 \mathrm{mmol}$, yield = 89\%). ${ }^{1} \mathrm{H}$ NMR (400 MHz, $\delta$ from TMS (ppm), $\left.\mathrm{CDCl}_{3}\right): \delta$ $0.89(\mathrm{t}, 3 \mathrm{H}), 1.38(\mathrm{~m}, 11 \mathrm{H}), 1.68(\mathrm{~m}, 2 \mathrm{H}), 3.94(\mathrm{~s}, 3 \mathrm{H}), 5.16(\mathrm{sext}, 1 \mathrm{H}), 7.27(\mathrm{~d}, 2 \mathrm{H})$, $8.09(\mathrm{~d}, 2 \mathrm{H}) .{ }^{13} \mathrm{C} \mathrm{NMR}\left(400 \mathrm{MHz}, \delta\right.$ from TMS (ppm), $\left.\mathrm{CDCl}_{3}\right): \delta 165.3,154.3,131.1$, $128.7,120.8,72.0,55.6,36.0,31.7,29.1,25.4,22.6,20.1,14.1 .[\alpha]_{589}^{\mathrm{RT}}=+3.00^{\circ}$ $(0.0344 \mathrm{~g} / \mathrm{mL}$; THF) for $96 \%$ ee.

\subsubsection{3.(S)-4-hydroxy-benzoic acid 1-methyl-heptyl ester (S)-3}


A solution of $(\boldsymbol{S})-\mathbf{2}(2.25 \mathrm{~g}, 7.3 \mathrm{mmol})$ in EtOH $(10 \mathrm{~mL})$ was added dropwise to $35 \%$ ammonia solution $(30 \mathrm{~mL})$ at room temperature. TLC analysis showed complete reaction after a period of $1 \mathrm{~h}$. After EtOH was removed using a rotary evaporator, the resulting solution was extracted with dichloromethane, and the organic phase was then dried over $\mathrm{MgSO}_{4}$ and filtered. The crude product was purified by a silica gel column (chloroform/ethyl acetate, 1:3 v/v) to give a colorless liquid $(1.65 \mathrm{~g}, 6.6 \mathrm{mmol}$, yield = $90 \%$ ), which was dried in vacuo. ${ }^{1} \mathrm{H}$ NMR (400 MHz, $\delta$ from TMS (ppm), $\mathrm{CDCl}_{3}$ ): $\delta$ $0.87(\mathrm{t}, 3 \mathrm{H}), 1.35(\mathrm{~m}, 11 \mathrm{H}), 1.65(\mathrm{~m}, 2 \mathrm{H}), 5.12$ (sext, 1H), $6.34(\mathrm{~s}, 1 \mathrm{H}), 6.88(\mathrm{~d}, 2 \mathrm{H})$, $7.95(\mathrm{~d}, 2 \mathrm{H}) .{ }^{13} \mathrm{C}$ NMR (400 MHz, $\delta$ from TMS (ppm), $\left.\mathrm{CDCl}_{3}\right): \delta 166.5,160.1,131.9$, $123.1,115.2,71.8,36.1,31.8,29.2,25.5,22.620 .1,14.1 .[\alpha]_{589}{ }^{\mathrm{RT}}=-4.13^{\mathrm{o}}(0.0166$ $\mathrm{g} / \mathrm{mL}$; THF) for $95 \%$ ee.

\subsubsection{4. (R)-4-hydroxy-benzoic acid 1-methyl-heptyl ester $(\boldsymbol{R})-3$}

This compound was prepared using the same method that was described for $(\boldsymbol{S})-\mathbf{3}$. Quantities used: (R)-2 (2.41 g, $7.8 \mathrm{mmol})$, EtOH (10 mL), ammonia (30 mL). Quantities yield: colorless liquid $(1.96 \mathrm{~g}, 7.8 \mathrm{mmol}$, yield $=99 \%) .{ }^{1} \mathrm{H} \mathrm{NMR}(400 \mathrm{MHz}, \delta$ from TMS (ppm), $\left.\mathrm{CDCl}_{3}\right): \delta 0.87(\mathrm{t}, 3 \mathrm{H}), 1.35(\mathrm{~m}, 11 \mathrm{H}), 1.66(\mathrm{~m}, 2 \mathrm{H}), 5.12$ (sext, 1H), 6.35 $(\mathrm{s}, 1 \mathrm{H}), 6.88(\mathrm{~d}, 2 \mathrm{H}), 7.95(\mathrm{~d}, 2 \mathrm{H}) .{ }^{13} \mathrm{C} \mathrm{NMR}\left(400 \mathrm{MHz}, \delta\right.$ from TMS (ppm), $\left.\mathrm{CDCl}_{3}\right)$ : $\delta 166.6$, 160.0, 131.9, 123.0, 115.2, 71.8, 36.1, 31.8, 29.2, 25.5, 22.7, 20.2, 14.1 . $[\alpha]_{589}{ }^{\mathrm{RT}}=+4.16^{\mathrm{o}}(0.0139 \mathrm{~g} / \mathrm{mL}$; THF $)$ for $93 \%$ ee.

\subsubsection{5. (S,S)-4-(1-methyl-heptyloxy)-benzoic acid 1-methyl-heptyl ester $(\boldsymbol{S}, \boldsymbol{S})-\mathbf{4}$}

This compound was prepared using the same method that was described for $(\boldsymbol{S})-\mathbf{2}$. Quantities used: triphenylphosphine (1.65 g, $6.3 \mathrm{mmol}),(R)-(-)$-2-octanol (0.68 g, 5.2 $\mathrm{mmol}),(S)-3(1.31 \mathrm{~g}, 5.2 \mathrm{mmol})$, diethyl azodicarboxylate $(2.92 \mathrm{~g}, 14.4 \mathrm{mmol})$ and dry THF $(20 \mathrm{~mL})$. The crude product was purified by a silica gel column (chloroform $/ n$-hexane, $2: 3 \mathrm{v} / \mathrm{v}$ ) to give a colorless oil (1.58 g, $4.3 \mathrm{mmol}$, yield $=83 \%$ ), which was dried in vacuo. ${ }^{1} \mathrm{H}$ NMR (400 MHz, $\delta$ from TMS (ppm), $\left.\mathrm{CDCl}_{3}\right): \delta 0.81(\mathrm{~m}$, $6 \mathrm{H}), 1.29(\mathrm{~m}, 22 \mathrm{H}), 1.51(\mathrm{~m}, 2 \mathrm{H}), 1.66(\mathrm{~m}, 2 \mathrm{H}), 4.37$ (sext, 1H), 5.05 (sext, 1H), 6.81 (d, 2H), $7.90(\mathrm{~d}, 2 \mathrm{H}) .{ }^{13} \mathrm{C} \mathrm{NMR}\left(400 \mathrm{MHz}, \delta\right.$ from TMS (ppm), $\left.\mathrm{CDCl}_{3}\right): \delta 166.1,162.0$, $131.5,123.0,115.0,74.0,71.3,36.4,36.2$, 31.8, 31.7,29.3, 29.2, 25.5, 25.4, 22.6, 20.2, 19.6, 14.1. (KBr disc): 2930, 2860, 1710, 1605, 1580, 1510, 1460, 1420, 1370, 1250, 1170, 1110, 1050, 940, 850 and 770. $[\alpha]_{589}{ }^{\mathrm{RT}}=+3.81^{\mathrm{o}}(0.0231 \mathrm{~g} / \mathrm{mL}$; THF). Calc. for $\mathrm{C}_{23} \mathrm{H}_{38} \mathrm{O}_{3}: \mathrm{C}, 76.2 ; \mathrm{H}, 10.56$. Found (\%): C, 75.99; H, 10.00 .

\subsubsection{6. $(R, R)-4-(1-m e t h y l-h e p t y l o x y)-b e n z o i c ~ a c i d ~ 1-m e t h y l-h e p t y l ~ e s t e r ~(R, R)-4$}


This compound was prepared using the same method that was described for $(\boldsymbol{S})-\mathbf{2}$. Quantities used: triphenylphosphine (2.21 g, $8.4 \mathrm{mmol}),(S)$-(+)-2-octanol (0.92 g, 7.0 $\mathrm{mmol}),(\boldsymbol{R})-3$ (1.75 g, $7.0 \mathrm{mmol})$, diethyl azodicarboxylate $(3.88 \mathrm{~g}, 7.7 \mathrm{mmol})$ and dry THF (20 mL). Quantities yield: colorless oil (1.93 g, $7.0 \mathrm{mmol}$, yield =76\%). ${ }^{1} \mathrm{H} \mathrm{NMR}$ (400 MHz, $\delta$ from TMS (ppm), $\left.\mathrm{CDCl}_{3}\right): \delta 0.88(\mathrm{~m}, 6 \mathrm{H}), 1.35(\mathrm{~m}, 22 \mathrm{H}), 1.61(\mathrm{~m}, 2 \mathrm{H})$, $1.73(\mathrm{~m}, 2 \mathrm{H}), 4.43$ (sext, 1H), 5.12 (sext, 1H), $6.88(\mathrm{~d}, 2 \mathrm{H}), 7.97(\mathrm{~d}, 2 \mathrm{H}) .{ }^{13} \mathrm{C}$ NMR $(400$ $\mathrm{MHz}, \delta$ from TMS (ppm), $\mathrm{CDCl}_{3}$ ): $\delta 166.1,162.0,131.5,122.9,115.0,74.0,71.3,36.4$, $36.2,31.8,31.7,29.3,29.2,25.5,25.4,22.6,20.2,19.6,14.1 . v / \mathrm{cm}^{-1}$ ( $\mathrm{KBr}$ disc): 2930, $2860,1710,1605,1580,1510,1460,1420,1370,1250,1170,1110,1050,940,850$ and 770. $[\alpha]_{589}{ }^{\mathrm{RT}}=-3.80^{\circ}(0.0232 \mathrm{~g} / \mathrm{mL}$; THF $)$. Calc. for $\mathrm{C}_{23} \mathrm{H}_{38} \mathrm{O}_{3}: \mathrm{C}, 76.2 ; \mathrm{H}, 10.56$. Found (\%): C, 75.79; H, 10.33 .

\section{Results and discussion}

\subsection{Synthesis}

Scheme 1 shows synthetic routes to an enantiomeric pair of chiral inducers with double chiral carbons. The target compounds were synthesized in three steps. First, methyl 4-carboxyphenyl carbonate (1) was esterified with $(S)$ - and $(R)$-2-octanol via Mitsunobu reaction, using diethyl azodicarboxylate and triphenylphosphine to give the $(R)$ - and $(S)$-protected esters $((\boldsymbol{R})-\mathbf{2}$ and $(\boldsymbol{S})-\mathbf{2})$ as colorless oils. Generally, Mitsunobu reaction occurs with inversion of stereo-configuration at asymmetric carbons attached to hydroxyl groups. Enantiomeric excess values of compound $(R)-2$ and $(S)-2$ were both 96\%. Compounds $(\boldsymbol{R})-\mathbf{2}$ and $(\boldsymbol{S})-\mathbf{2}$ were deprotected using an ethanol-ammonia solution at room temperature to give compounds $(\boldsymbol{R})-\mathbf{3}$ and $(\boldsymbol{S})-\mathbf{3}$ in good yield. Enantiomeric excess values of compounds $(\boldsymbol{R})-\mathbf{3}$ and $(\boldsymbol{S})-\mathbf{3}$ were $95 \%$ and $93 \%$, respectively. Finally, compounds $(\boldsymbol{R}, \boldsymbol{R}) \mathbf{- 4}$ and $(\boldsymbol{S}, \boldsymbol{S}) \mathbf{- 4}$ were synthesized by same procedure as for compounds $(\boldsymbol{R})-2$ and $(\boldsymbol{S})-2$. Compound $(\boldsymbol{R})-4$ displayed sextet peaks at 4.43 and $5.11 \mathrm{ppm}$ from TMS in the NMR spectra respectively, attributable to a proton attached to the stereogenic centers, and two signals in the low magnetic region due to protons attached to the benzene ring (Fig. S1). Electrochemical synthesis of poly(TBT) were performed in CLC phase induced by the addition of $(\boldsymbol{R}, \boldsymbol{R})-\mathbf{4}$ or $(\boldsymbol{S}, \boldsymbol{S})-\mathbf{4}$ as chiral inducers.

\subsection{Optical Rotation and Helical Twisting Power}

Table 1 shows the optical rotation and helical twisting power of $(\boldsymbol{R})-\mathbf{2},(S)-\mathbf{2},(\boldsymbol{R})-\mathbf{3}$, $(S)-3,(R, R)-4$ and $(S, S)-4$. The sign of the specific rotation of $(R, R)-4$ changed from (-) to $(+)$ with inversion of the configuration. On the other hand, $(S, S)-4$ changed from $(+)$ to $(-)$. This results demonstrated that the Mitsunobu reaction proceeded with inversion 
of configuration. When a chiral inducer is added to a nematic LC, the LC molecules form a twisted structure due to sergeant-soldier effect. The ability of the chiral inducer to twist the achiral nematic phase is expressed as an HTP. The HTP of $(\boldsymbol{R}, \boldsymbol{R})-\mathbf{4}$ and $(S, S)-4$ were studied in solutions of CLC which was induced by addition of $(\boldsymbol{R}, \boldsymbol{R})-\mathbf{4}$ and $(S, S)-4(0.02-0.03$ fractions) to nematic 6CB. Furthermore, the HTP is estimated by the equation: $\beta=(\mathrm{pc})^{-1}$ where $\beta\left(\mu \mathrm{m}^{-1}\right)$ is HTP, $\mathrm{p}(\mu \mathrm{m})$ is the helical pitch of the CLC phase, and $\mathrm{c}(\mathrm{wt} \%)$ is the concentration of the chiral inducer in the CLC. The value of $\beta$ was measured by the Grandjean-Cano wedge method ( $\tan \theta=0.0083)$, which was based on the observation of the distance between discontinuity lines. The disclination lines of the CLC in the wedge cell were observed through a polarizing optical microscopy (POM) (Fig. S5). The values of $(\boldsymbol{R})-\mathbf{2}$ and $(\boldsymbol{S})$-2 were higher than for $(\boldsymbol{R})-\mathbf{4}$ and $(\boldsymbol{S}) \mathbf{- 4}$, respectively. The magnitude of HTP is largely influenced by the linkages between chiral center and direction of the polar group. These results demonstrated that a balance of steric effect and polar group influence is important to design chiral inducers with high HTP.

\subsection{Preparation of CLC electrolyte solution and determination of the helical direction}

A CLC electrolyte solution was prepared by the addition of $(\boldsymbol{R}, \boldsymbol{R})-\mathbf{4}$ or $(\boldsymbol{S}, \boldsymbol{S})-\mathbf{4}$ as chiral inducers, along with TBAP as a supporting salt, to $6 \mathrm{CB}$ and $8 \mathrm{OCB}$ mixture as the NLC solvent. TBT was dissolved in the electrolyte solutions as a monomer. The molecular structure and composition of the CLC electrolyte are shown in Table 2. The electrolyte solution prepared exhibited a CLC phase texture at room temperature. The electrolyte solution displayed a fingerprint texture under POM observation (Fig. 1 (a), (c)). A helical sense of $(R)$-CLC and $(S)$-CLC was also determined by a miscibility test using cholesteryl oleyl carbonate (COC) as a standard CLC (Fig. S6). The helical direction of the COC is known to be left-handed. The miscibility test is based on the observation of the mixing area between the CLC and the standard CLC using POM. If the helical sense is the same as the standard CLC, the observation of the contact area is continuous. On the contrary, the helical direction of the contact area is discontinuous when a test sample has opposite helical direction against a standard LC. Fig. S6 shows the POM images of the miscibility test of $(\boldsymbol{R}, \boldsymbol{R})-\mathbf{4}$ and $(\boldsymbol{S}, \boldsymbol{S})-\mathbf{4}$ with COC as a standard. These observations suggested that the helical direction of $(R)$-CLC was right-handed and (S)-CLC was left-handed.

\subsection{Electrochemical polymerization in CLC}

The CLC electrolyte was injected between sandwiched indium-tin-oxide (ITO) coated 
electrodes ( $9 \Omega \mathrm{cm}^{-2}$, surface area, $1 \mathrm{~cm}$ x $5 \mathrm{~cm}$, thickness, ca. $12 \mathrm{~nm}$ ) using a Teflon sheet (thickness, ca $0.2 \mathrm{~mm}$ ) as a spacer [20]. The POM image confirmed the CLC phase of the electrolyte solution at room temperature. Polymerization was performed with a constant 4.0 V DC voltage applied to the sandwiched cell. The application of this polymerization voltage did not affect the optical texture of the CLC electrolyte. However, repeated voltage scanning for cyclic voltammetry was found to destroy the helical structure [20]. After reaction for $30 \mathrm{~min}$, the residual CLC mixture was washed off with hexane providing a thin film, $(\boldsymbol{R})-\mathbf{P T B T}_{\mathbf{0 T}}$ and $(\boldsymbol{S})-\mathbf{P T B T}_{\mathbf{0 T}}$ (polymerized under zero magnetic field) on the anode side of the ITO electrode. Furthermore, electrochemical polymerization of TBT under magnetic field of $4 \mathrm{~T}$ was carried out with the CLC electrolyte at room temperature. The reaction cell was heated to obtain isotropic phase and then quickly placed in a drum type magnetic chamber at room temperature. Magnetic alignment was carried out for $30 \mathrm{~min}$ in the magnetic field of $4 \mathrm{~T}$ before polymerization. Then, the constant DC voltage of $4.0 \mathrm{~V}$ was applied to the cell for $30 \mathrm{~min}$ to perform electrochemical polymerization. After the reaction, the polymer film on an anodic surface was washed with hexane to remove LC electrolyte solution and oligomers, which provided $(\boldsymbol{R})-\mathbf{P T B T}_{\mathbf{4 T}}$ and $(\boldsymbol{S})-\mathbf{P T B T}_{\mathbf{4 T}}$.

\subsection{Polymer characterization}

The polymers were obtained as insoluble and infusible films on ITO glass electrodes. According to the DFT calculation results, electrochemical polymerization reaction is based on a radical coupling reaction between the $\alpha$-positions of the thiophene rings (Fig. S4). Surface texture of the polymer films were confirmed by POM. (R)-PTBT $\mathbf{P T}_{\mathbf{0 T}}$ and (S)-PTBT ${ }_{\text {0T }}$ exhibited fingerprint texture, which is similar to that of the CLC electrolyte solution (Fig. 1 (a)-(d)). The fingerprint texture is derived from the helicity of LC molecules. This suggested that the polymer chains propagated along the LC molecules, leading to the transcription of the helical structure of the CLC. In contrast, (R)-PTBT ${ }_{4 \mathbf{T}}$ and $(\boldsymbol{S})$-PTBT $_{4 \mathbf{T}}$ displayed a stripe texture (Fig. 1 (e), (f)). The direction of stripes was parallel to the magnetic field. The stripes were derived from the helical periodic structure of the CLC which indicated that the helical axis was vertically aligned with the magnetic field. Furthermore, LD spectra of $(\boldsymbol{R})-\mathbf{P T B T}_{\mathbf{4 T}}$ show slightly red shift and negative LD as applied voltage increases (Fig. S8). The spectra suggest that the chromophore of the polymer is aligned. These results further indicated that the molecular arrangement of the polymers was achieved by the application of magnetic field. 


\subsection{Optical Properties}

The CD and UV-vis absorption spectra of $(\boldsymbol{R})-\mathbf{P T B T}_{\mathbf{0 T}}$ and $(\boldsymbol{S})-\mathbf{P T B T}_{\mathbf{0 T}}$ were obtained under application of voltages in an electrochemical method in a monomer-free $0.1 \mathrm{M}$ TBAP-acetonitrile solution (Fig. 2-4). The optical band gap $\left(E_{\mathrm{g}}\right)$ of $(\boldsymbol{R})-\mathbf{P T B T}_{\mathbf{0 T}}$ is estimated from the low energy edge $(537 \mathrm{~nm})$ of the absorption spectrum. The $E_{\mathrm{g}}$ of $(\boldsymbol{R})-$ PTBT $_{\text {oT }}(2.31 \mathrm{eV})$ is consistent with poly(TBT), which was electrochemically synthesized in acetonitrile solution [21]. The polymers showed a reversible change in the absorption intensity at $600 \mathrm{~nm}\left(\pi-\pi^{*}\right.$ transition of main chain) and at $900 \mathrm{~nm}$ (polaron band). In the $\mathrm{CD}$, the polymers show bisignate Cotton effect near their absorption maximum wavelengths. This type of bisignate Cotton effect indicating chiral aggregation of the polymer backbone [22]. (R)-PTBT $\mathbf{P T}_{\mathbf{0 T}}$ show first positive and second negative Cotton effect, indicating that the polymer back bones form right-handed helical orientation. This right-handed helical orientation is consistent with the right-handed helical order of $(S)$-CLC electrolyte solution. On the contrary, $(\boldsymbol{S})$-PTBT $\mathbf{P T}_{\mathbf{0 T}}$ shows first negative and second positive Cotton effect, indicating left-handed helical orientation of the polymer backbone. These results suggested that the chiroptical activity of the polymers could be controlled by using $(\boldsymbol{R}, \boldsymbol{R})-\mathbf{4}$ and $(\boldsymbol{S}, \boldsymbol{S})-\mathbf{4}$ as chiral inducers.

The magnitude and absorption maximum of $C D$ and $U V-$ vis spectra for the polymers were changed by the oxidation and reduction processes, which were due to the structural change in the main chain. The electrochemical oxidation process produced polarons and bipolarons on the main chain accompanied by structural change from benzenoid structure to planar quinoid structure, resulting in an increase of coplanarity and rigidity of the main chain. The polymers exhibited intense CD signals in the reduced state, and weak signals in the oxidized state. The color of the polymers also drastically changed from dark-green to light-green during the reduced process. Therefore, the optical activity and absorption characteristics of the polymers were tunable by the application of voltage, which may be potentially useful for electrochromic applications.

\section{Conclusions}

A series of chiral inducers having double stereogenic centers were successfully synthesized via Mitsunobu reaction. A CLC was prepared through the addition of a small amount of $(\boldsymbol{R}, \boldsymbol{R})-\mathbf{4}$ and $(\boldsymbol{S}, \boldsymbol{S})-\mathbf{4}$ as a chiral dopant into a NLC. The CLC electrolyte enabled the synthesis of chiroptically active polymers by electrochemical polymerization. Furthermore, the helical direction of the polymers was consistent with the helical order of the CLC electrolyte solution. The polymer film prepared under magnetic field of $4 \mathrm{~T}$ displayed a parallel stripe texture and exhibited liner dichroism 
due to the orientation of CLC electrolyte solution matrix. This result suggests that the chiral inducer shows good affinity to the LC molecules. The electrochemical polymerization using CLC as an electrolyte solution is expected to be applicable for the preparation of a range of new functional conjugated polymer materials with useful chiroptical properties. Moreover, the morphology of the polymer can easily be controlled by combining electrochemical polymerization in the CLC electrolyte and magnetic field. The research of chiral inducers opens further possibilities for chiroptical LC materials.

\section{Acknowledgements}

We would like to thank Chemical Analysis Center of University of Tsukuba and Glass Work Shop of University of Tsukuba. This research was supported by National Institute for Materials Science (NIMS), Japan.

\section{References}

[1] C. K. Chiang, C. R. Fincher, Y. W. Park, A. J. Heeger, H. Shirakawa, E. J. Louis, S.

C. Gau, A. G. MacDiarmid, Phys. Rev. Lett. 39 (1977) 1098-1101.

[2] C. E. D. Chidsey, R. W. Murray, Science 231 (1986) 25-31.

[3] D. S. K. Mudlgonda, J. L. Boehme, T. D. Brotherston, D. L. Meeker, J. P. Ferrarts, Chem. Mater. 12 (2000) 1508-1509.

[4] B. N. Boden, K. J. Jardine, A. C. W. Leung, M. J. MacLachlan, Org. Lett. 8 (2006) 1855-1858.

[5] G. Nystrom, A. Razaq, M. Stromme, L. Nyholm, Nano Lett. 9 (2009) 3635-3639.

[6] M. S. Wrighton, Science 231 (1986) 32-37.

[7] V. C. Goncalves, D. T. Balogh, Sens. Actuatators. B 162 (2012) 307-312.

[8] P. Novak, K. Muller, K. S. V. Santhanam, O. Haas, Chem. Rev. 97 (1997) 207-281.

[9] W. S. Schlindwein, Y. Gofer, H. Sarker, T. O. Poehler, P. C. Searson, J. Electroanal. Chem. 460 (1999) 46-52.

[10] H. Goto, K. Akagi, Synth. Met. 119 (2001) 165-166.

[11] H. Nakashima, M. Fujiki, J. R. Koe, M. Motonaga, J. Am. Chem. Soc. 123 (2001) 1963-1969.

[12] M. Ishikawa, K. Maeda, Y. Mitsutsuji, E. Yashima, J. Am. Chem. Soc. 126 (2004) 732-733.

[13] T. Nakano, J. Chromatography A 906 (2001) 205-225.

[14] K. Kawabata, H. Yoneyama, H. Goto, Mol. Cryst. Liq. Cryst. 515 (2009) 3-15.

[15] H. Goto, S. Nimori, J. Mater. Chem. 20 (2010) 1891-1898. 
[16] O. Mitsunobu, Synthesis (1981) 1-28.

[17] Y. Wang, Q. Li, Adv. Mater. 24 (2012) 1926-1945.

[18] A. Marini, V. Domenici, C. Malanga, R. Menicagli, C. A. Veracini, Tetrahedron 66 (2010) 3472-3477.

[19] M. Hemgesberg, D. M. Ohlmann, Y. Schmitt, M. R. Wolfe, M. K. Muller, B. Erb, Y. Sun, L. J. Gooben, M. Gerhardes, W. R. Thiel, Euro. J. Org. Chem. 11, (2012) 2142-2151.

[20] H. Yoneyama, A. Tsujimoto, and H. Goto, Macromolecules 40 (2007) 5279-5283.

[21] L. X. J. Zhao, C. C. R. Liu, J. L. H. Wang, Electrochim. Acta 56 (2011) 2815-2822.

[22] B. M. W. Langeveld-Voss, M. P. T. Christiaans, R. A. J. Janssen, E. W. Meijer, Macromolecules 31 (1998) 6702-6704. 
Table 1 Optical rotation and helical twisting power of $(R)-2,(S)-2,(R)-3,(S)-3,(R, R)-4$ and $(S, S)-4$.

Specific rotation for isolated molecule

$\left(\mathrm{deg} \cdot \mathrm{cm}^{2} / \mathrm{g}\right)^{a}$
Helical twisting power

$$
\beta\left(\mu \mathrm{m}^{-1}\right)^{b}
$$

$(\boldsymbol{R})-2$

$(S)-2$

$(\boldsymbol{R})-\mathbf{3}$

(S)-3

$(R, R)-4$

$(S, S)-4$
$-3.21$

$+3.00$

$-4.13$

$+4.16$

$+3.81$

6.07

$-3.80$

6.00

8.45

8.79
${ }^{a}$ The experimental values of $[\alpha]_{589}$ were obtained in tetrahydrofuran (THF) solution. ${ }^{b}$ In $6 \mathrm{CB}$. 
Synthesis of chiral inducers having double stereogenic centers for electrochemical polymerization in cholesteric liquid crystal medium

Synthetic Metals, 187, 217-223 (2014)

Table 2. Constituents of (R)-CLC and $(S)$-CLC electrolyte reaction solution.

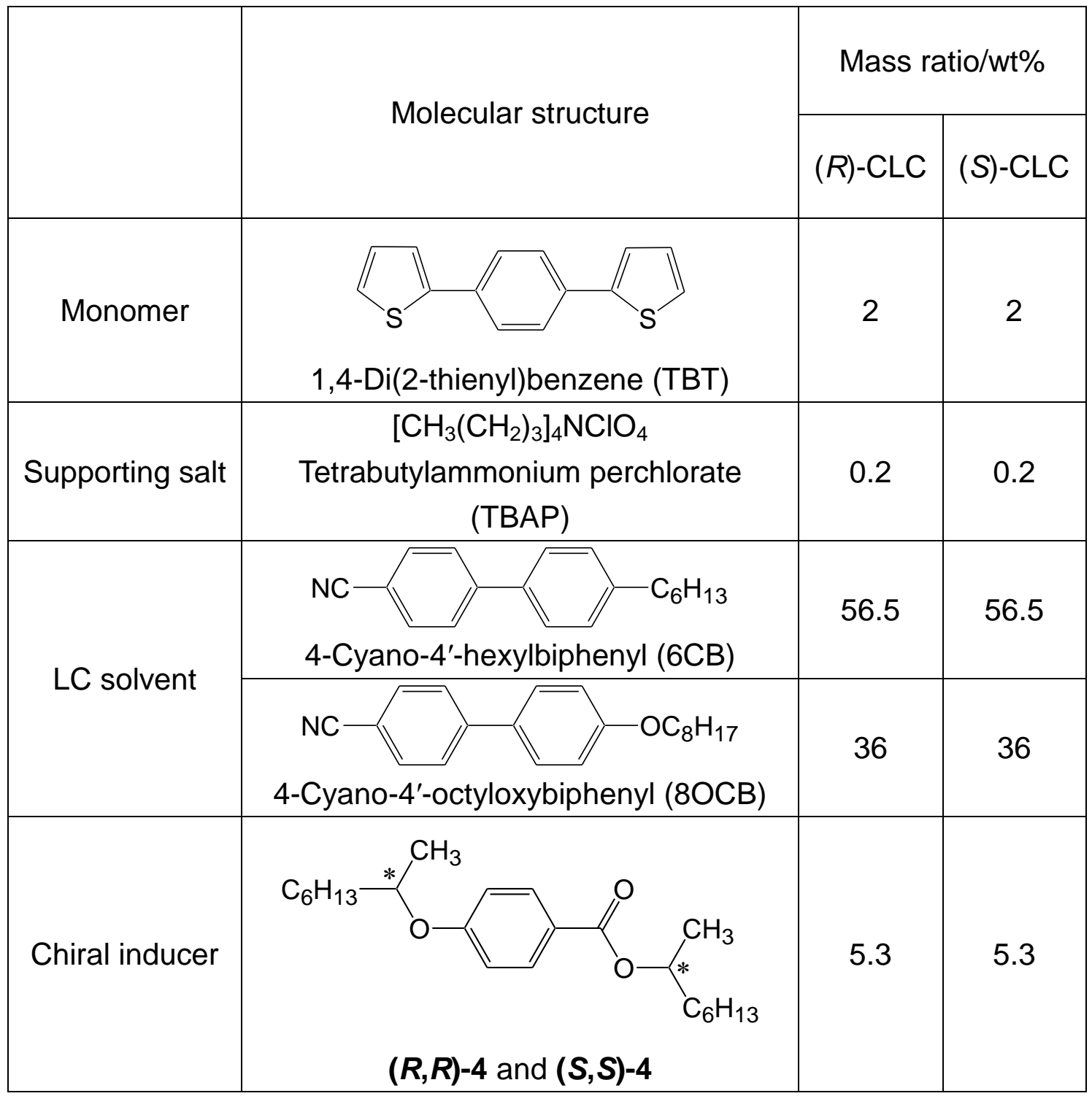



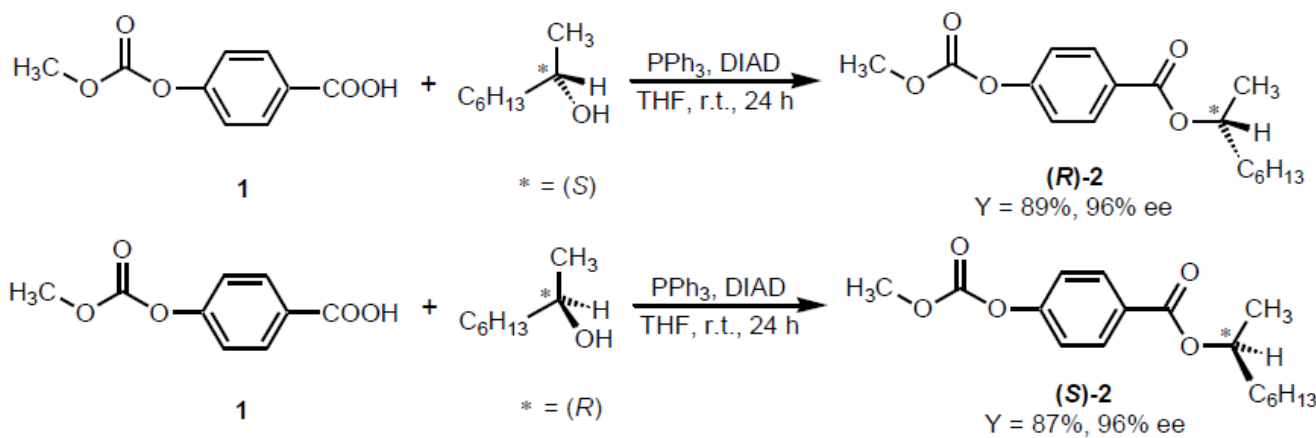

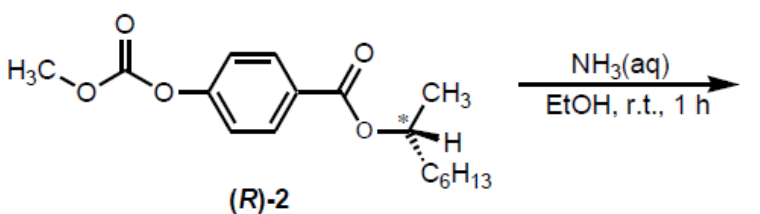

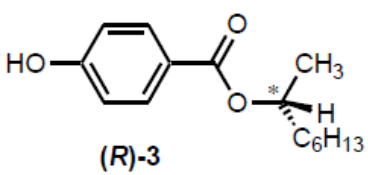

$Y=99 \%, 95 \%$ ee

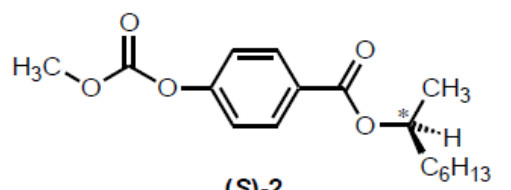

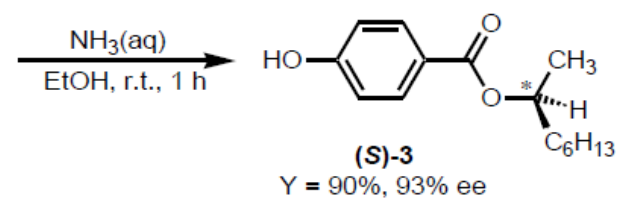

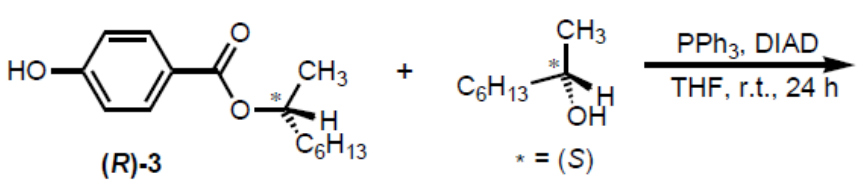

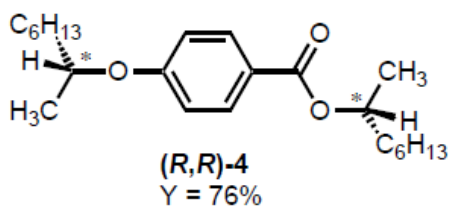

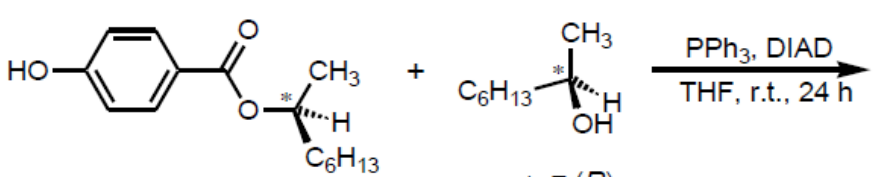

(S)-3 * $=(R)$

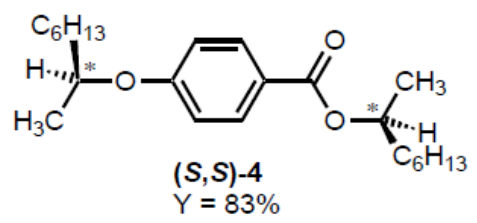

Scheme 1. Synthetic routes for $(\boldsymbol{R}, \boldsymbol{R})-4$ and $(S, S)-4,4-(1-m e t h y l-h e p t y l o x y)-b e n z o i c$ acid 1-methyl-heptyl esters.
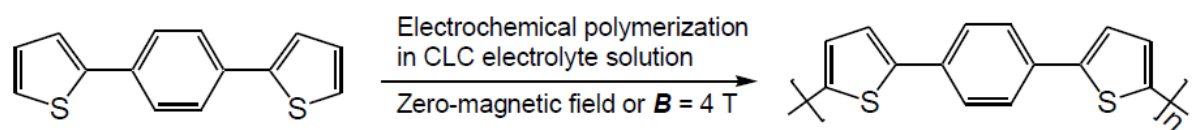

(R)-PTBT

(S)-PTBT

Scheme 2. Asymmetric electrochemical syntheses of $(\boldsymbol{R})$-PTBT and $(\boldsymbol{S})$-PTBT. 


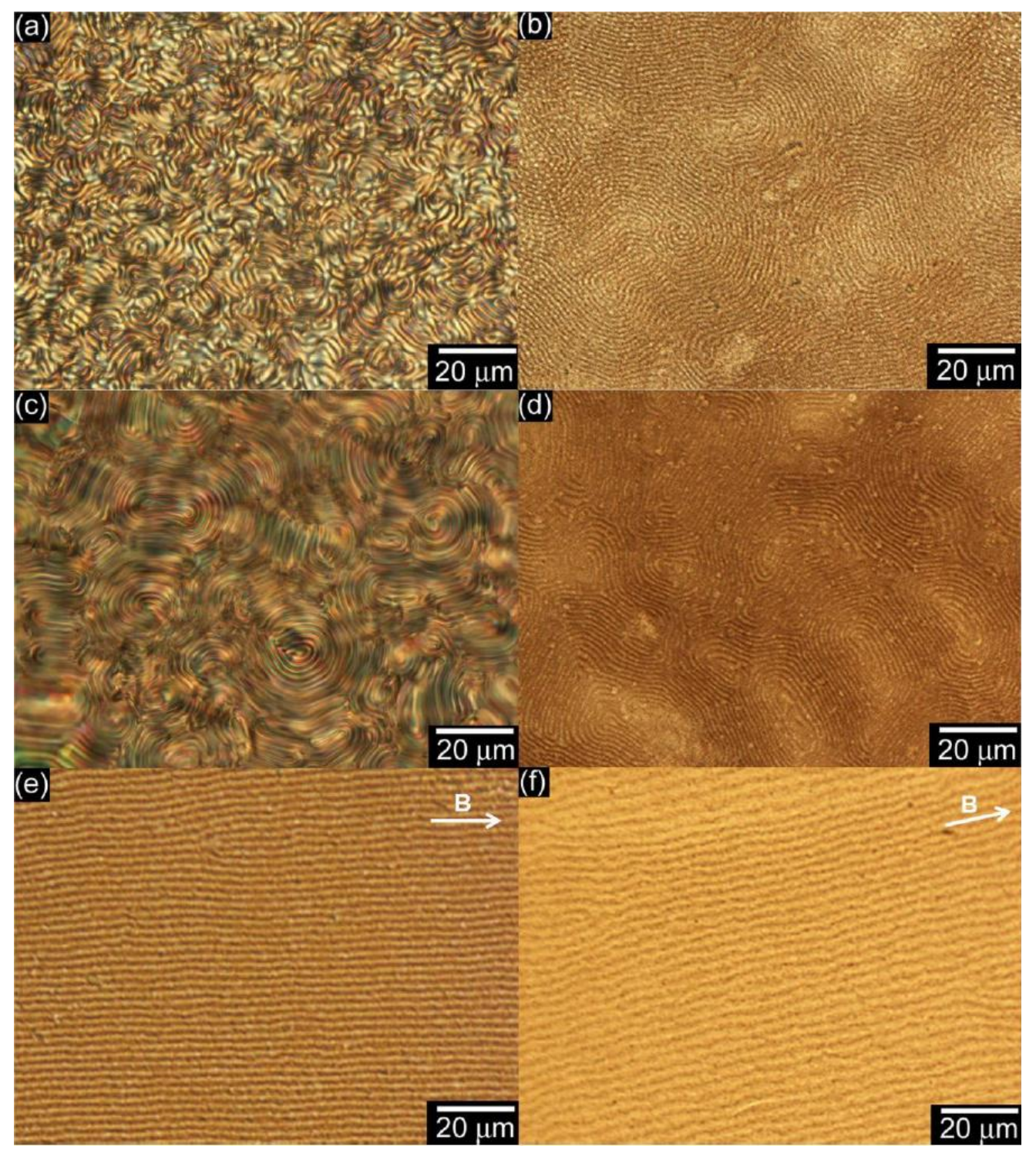

Fig. 1. (a) Polarizing optical microscopy (POM) image of (R)-CLC electrolyte solution containing the monomer at rt. (b) POM image of PTBT $_{\mathbf{0 T}}$ synthesized in (R)-CLC electrolyte solution $\left((\boldsymbol{R})-\mathbf{P T B T}_{\mathbf{0 T}}\right)$. (c) POM image of (S)-CLC electrolyte solution containing the monomer at rt. (d) POM image of PTBT $_{\mathbf{0 T}}$ synthesized in (S)-CLC electrolyte solution $\left((\boldsymbol{S})\right.$-PTBT $\left.\mathbf{P T}_{\mathbf{0 T}}\right)$. (e) POM image of PTBT $_{\mathbf{4 T}}$ synthesized in (R)-CLC electrolyte solution under magnetic field $\left((\boldsymbol{R})-\mathbf{P T B T}_{4 \mathbf{T}}\right)$. (f) POM image of $\mathbf{P T B T}_{4 \mathbf{T}}$ synthesized in $(S)$-CLC electrolyte solution under magnetic field $\left((S)-\mathbf{P T B T}_{4 \mathbf{T}}\right)$. 
Synthesis of chiral inducers having double stereogenic centers for electrochemical polymerization in cholesteric liquid crystal medium
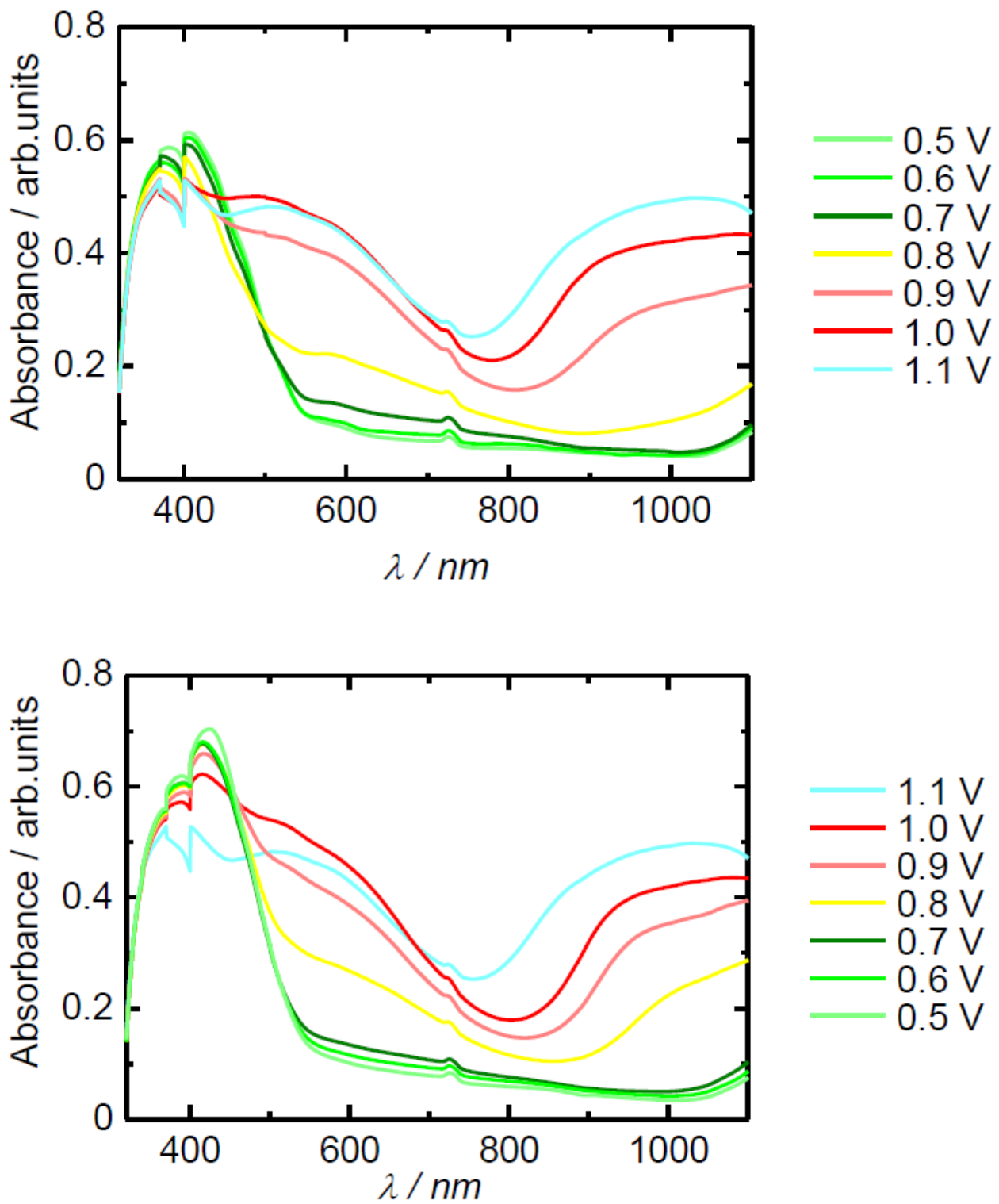

Fig. 2. Optical absorption spectra of $(\boldsymbol{R})-\mathbf{P T B T}_{\mathbf{0 T}}$ at various applied potentials vs. $\mathrm{Ag} / \mathrm{Ag}^{+}$reference electrode during oxidation process (upper) and reduction process (lower). 
Synthesis of chiral inducers having double stereogenic centers for electrochemical polymerization in cholesteric liquid crystal medium
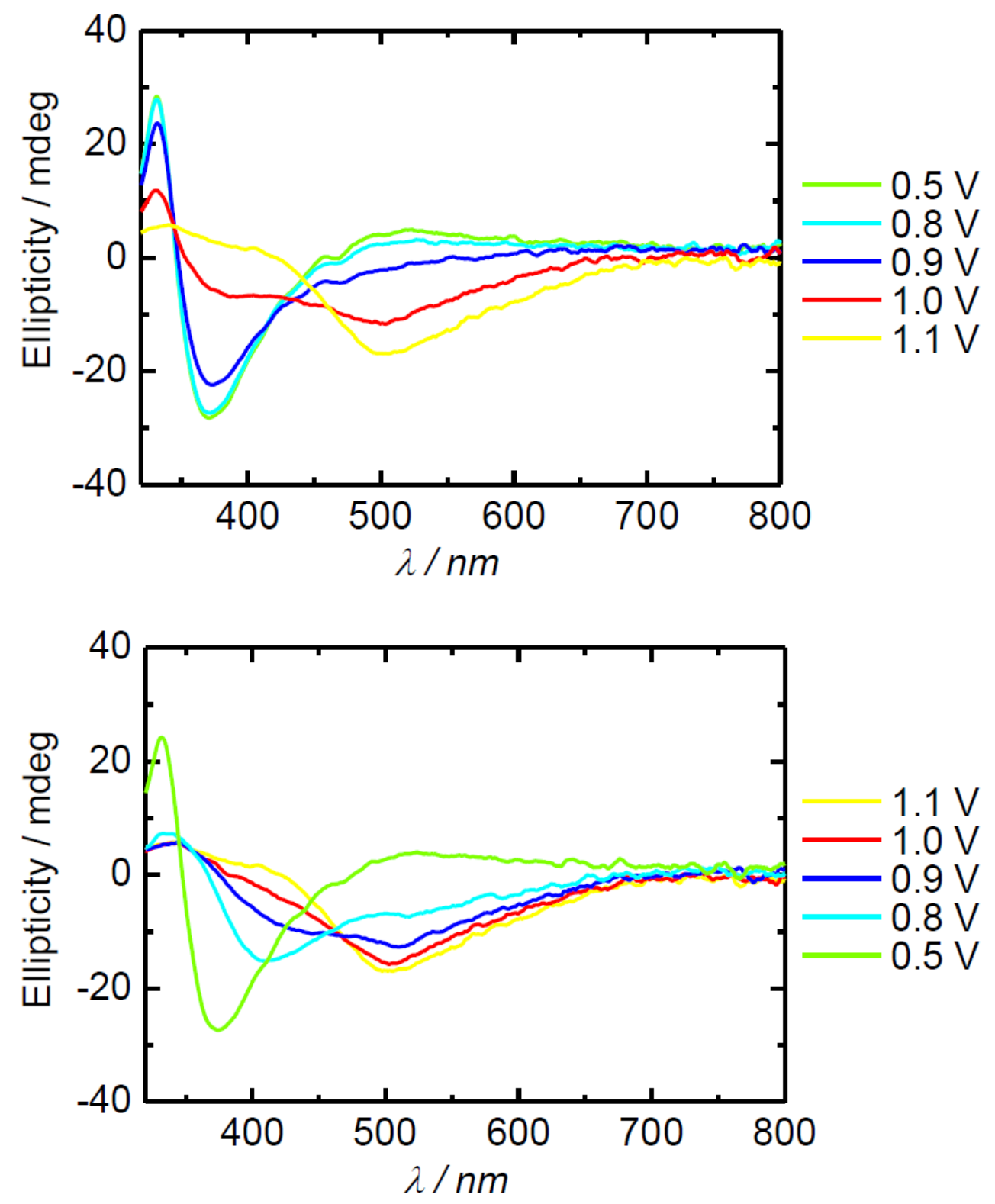

Fig. 3. CD spectra of $(\boldsymbol{R})-\mathbf{P T B T}_{\mathbf{0 T}}$ at various applied potentials vs. $\mathrm{Ag} / \mathrm{Ag}^{+}$reference electrode during oxidation process (upper) and reduction process (lower). 
Synthesis of chiral inducers having double stereogenic centers for electrochemical polymerization in cholesteric liquid crystal medium
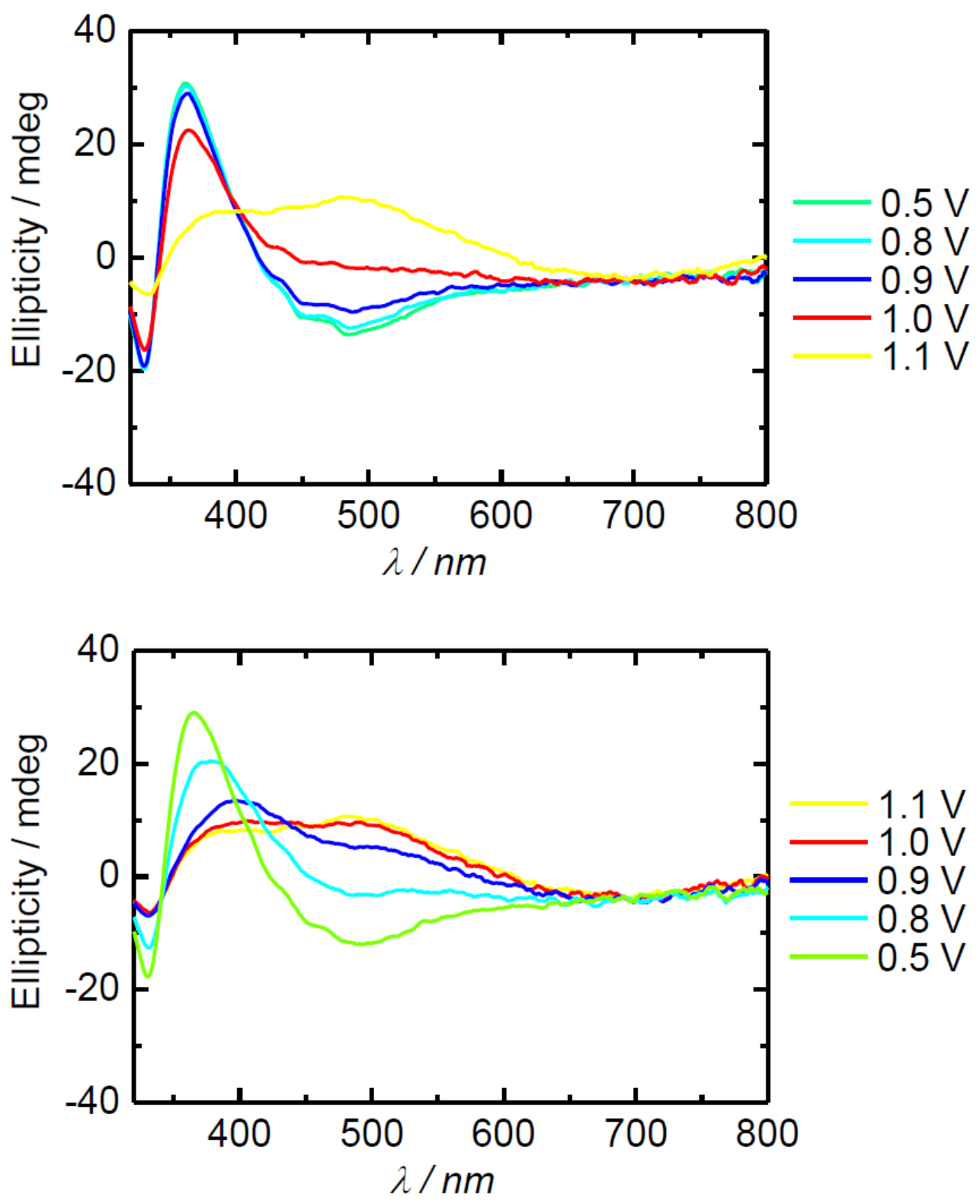

Fig. 4. CD spectra of $(\boldsymbol{S})-\mathbf{P T B T}_{\mathbf{0 T}}$ at various applied potentials vs. $\mathrm{Ag} / \mathrm{Ag}^{+}$reference electrode during oxidation process (upper) and reduction process (lower). 


\section{Supporting Information for:}

Synthesis of chiral inducers having double stereogenic centers for electrochemical polymerization in cholesteric liquid crystal medium

Tomokazu Iseki, ${ }^{1}$ Kohsuke Kawabata, ${ }^{1}$ Shigeki Nimori, ${ }^{2}$ Hiromasa Goto ${ }^{1 *}$

${ }^{1}$ Division of Materials Science, Faculty of Pure and Applied Sciences, University of Tsukuba, Tsukuba, Ibaraki 305-8573, Japan

${ }^{2}$ Tsukuba Magnet Laboratory, National Institute for Materials Science (NIMS), Tsukuba, Ibaraki 305-0003, Japan

Correspondence to H. Goto, e-mail: gotoh@ims.tsukuba.ac.jp, fax: +81-29-853-4490 
Iseki, T; Kawabata, K; Nimori, S; Goto, $\mathrm{H}^{*}$

Synthesis of chiral inducers having double stereogenic centers for electrochemical polymerization in cholesteric liquid crystal medium

Synthetic Metals, 187, 217-223 (2014)

\section{${ }^{1}$ H NMR spectra}

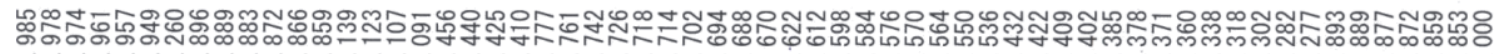
-

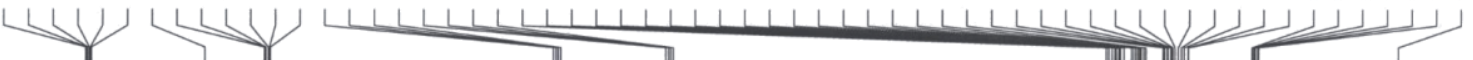

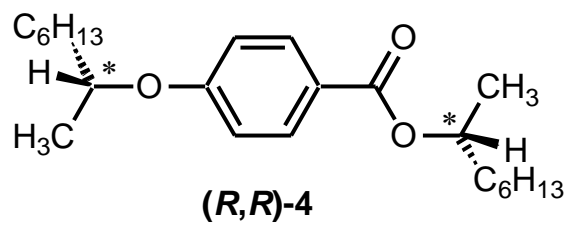

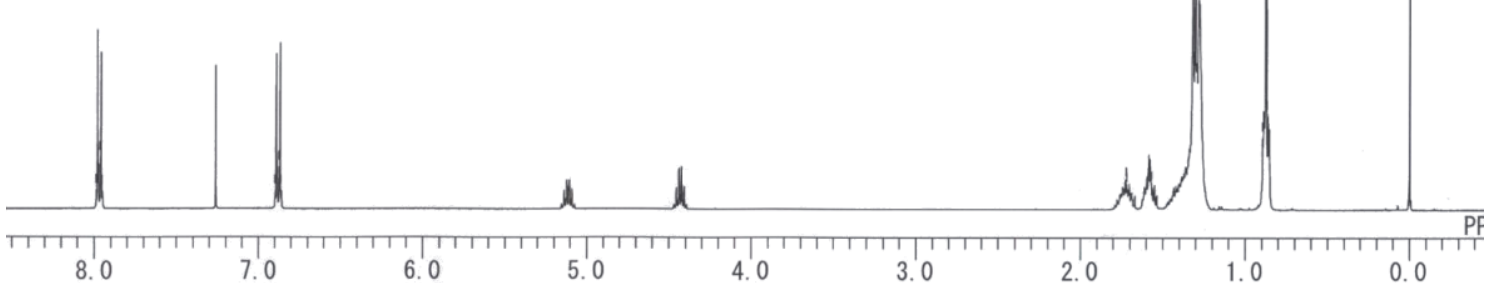

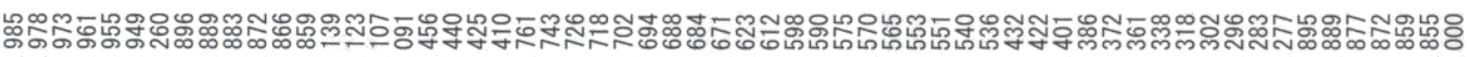

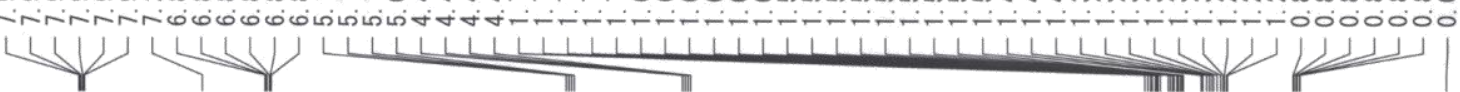
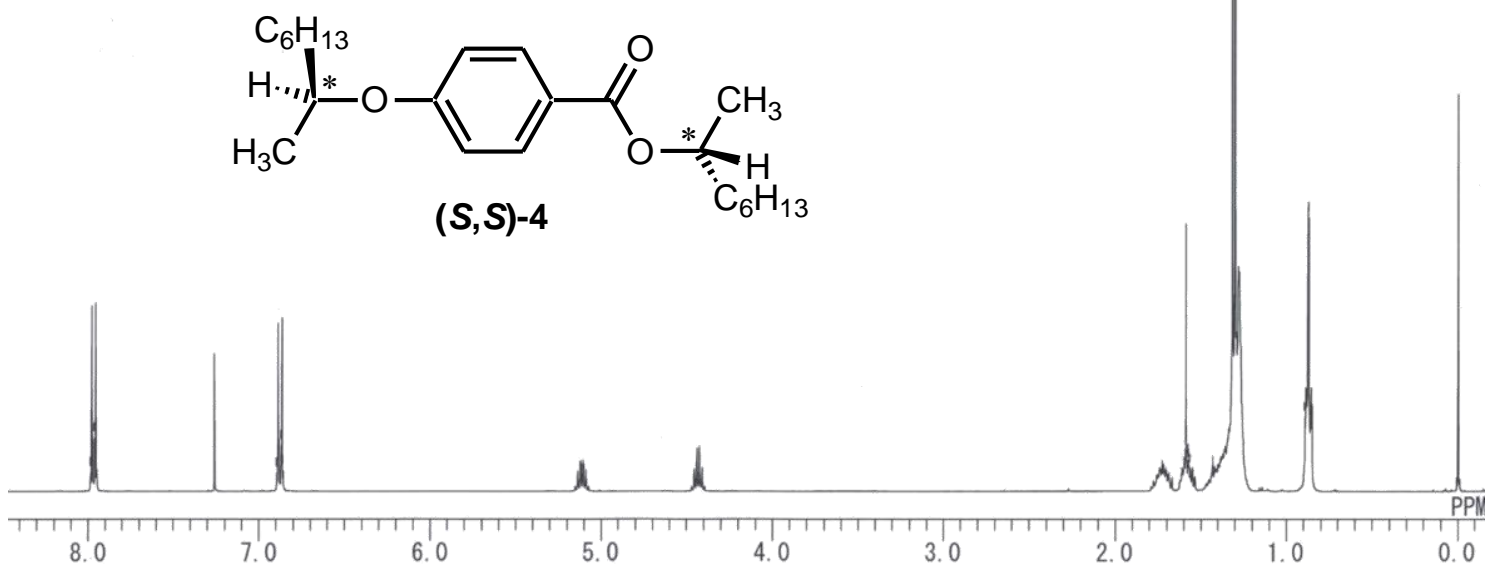

Fig. S1. ${ }^{1} \mathrm{H}$ NMR spectra of compound $(\boldsymbol{R}, \boldsymbol{R})-\mathbf{4}$ (upper) and $(\boldsymbol{S}, \boldsymbol{S})-\mathbf{4}$ (lower). 


\section{IR spectra}

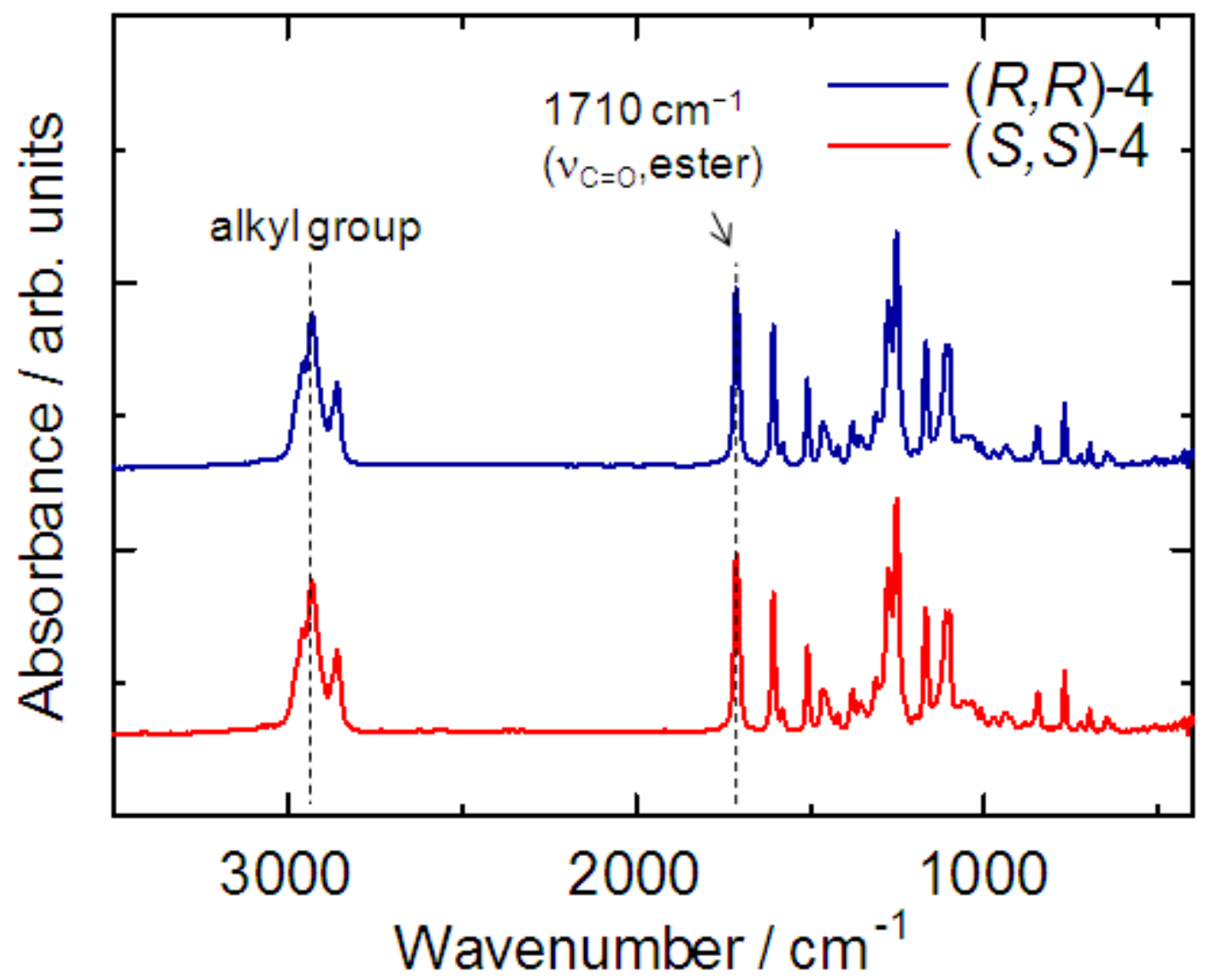

Fig. S2. IR absorption spectra of compound $(\boldsymbol{R}, \boldsymbol{R})-4$ (blue line) and $(S, S)-4$ (red line). 
Synthesis of chiral inducers having double stereogenic centers for electrochemical polymerization in cholesteric liquid crystal medium

CD spectra

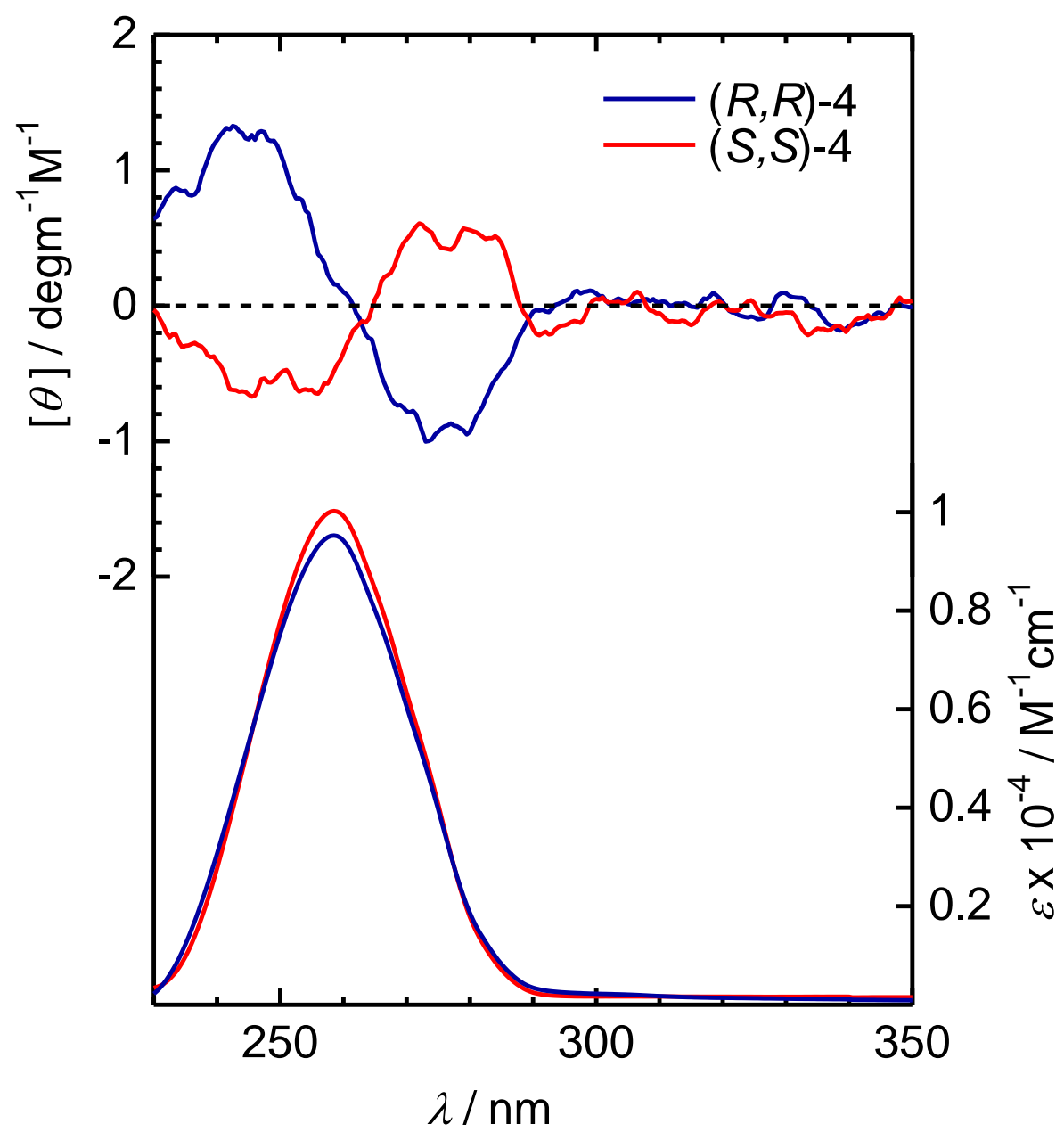

Fig. S3. CD spectra (upper) and UV-vis absorption spectra (lower) for $(\boldsymbol{R}, \boldsymbol{R})-\mathbf{4}$ (blue line) and $(S, S)-4$ (red line) in hexane. 


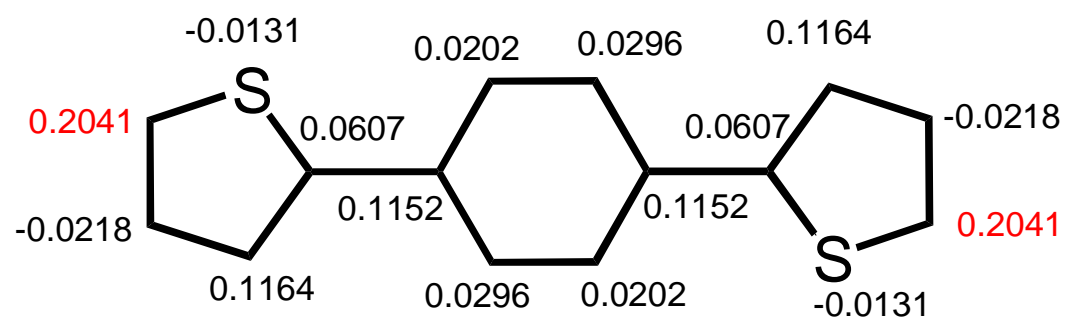

Fig. S4. Spin density on each atom of TBT in the radical cationic state calculated by DFT method (B3LYP-6-31G*) (Red colored sites indicate the most reactive sites. Hydrogen atoms are omitted for simplification.)

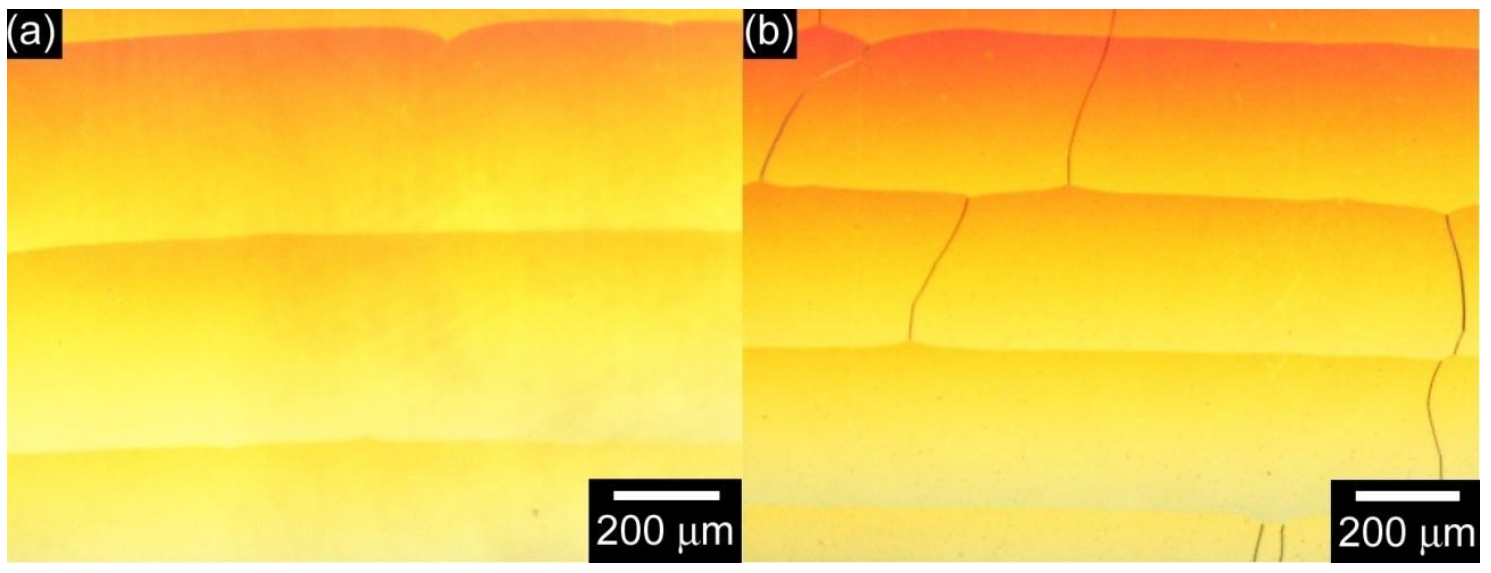

Fig. S5. POM images of the Cano-wedge cell containing the sample CLC. A nematic host is 6CB and chiral inducer is (a) $(\boldsymbol{R}, \boldsymbol{R})-\mathbf{4}$ and (b) $(\boldsymbol{S}, \boldsymbol{S})-\mathbf{4}$. 


\section{Contact test}

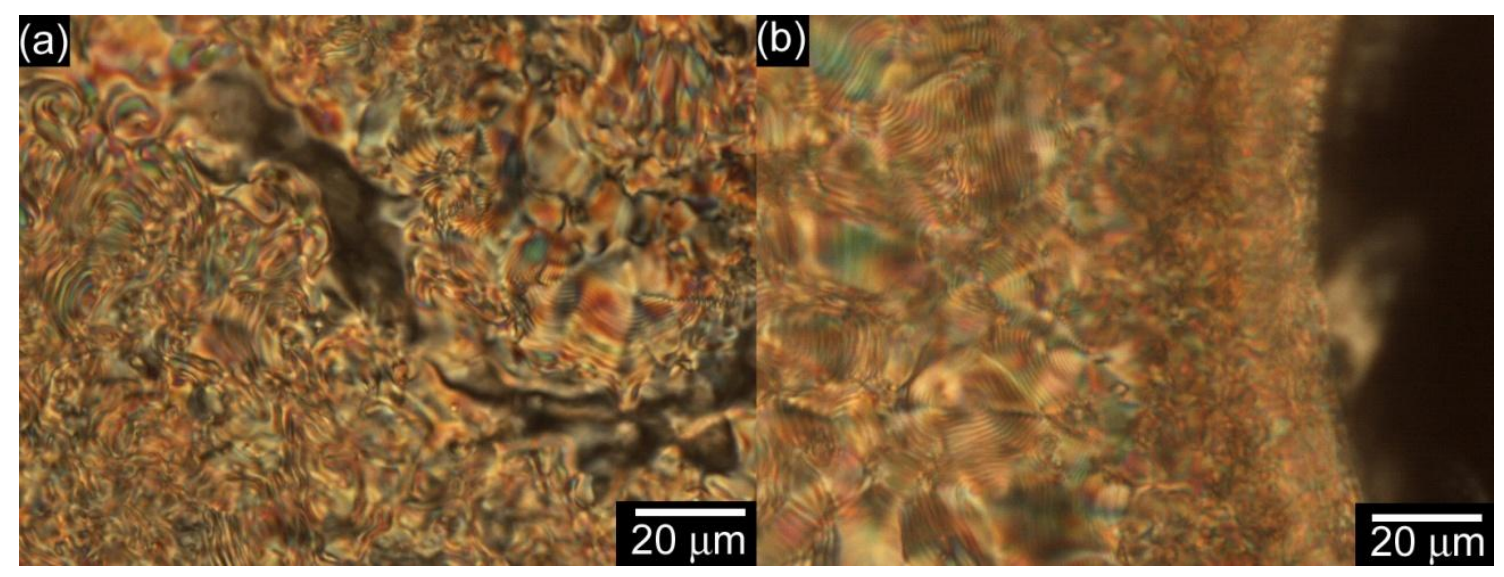

Fig. S6. POM images of contact tests between the CLC containing (a) $(\boldsymbol{R}, \boldsymbol{R})-\mathbf{4}$ or (b) $(S, S)-4$ and cholesteryl oreyl carbonate with a left-handed helical direction as standard.

Cyclic voltammogram

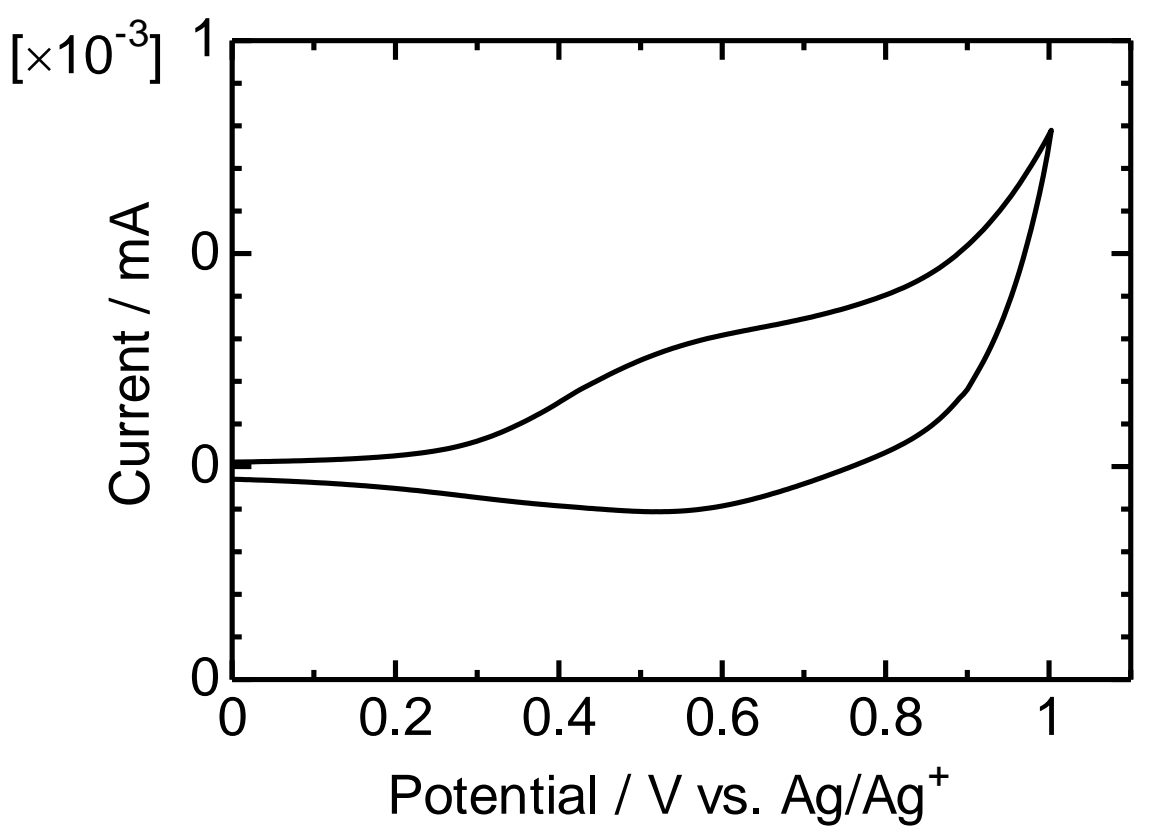

Fig. S7. Cyclic voltammogram of $(\boldsymbol{R})-\mathrm{PTBT}_{\mathbf{0 T}}$ on an ITO electrode in monomer-free 0.1 M TBAP/acetonitrile solution (scan rate: $100 \mathrm{mVs}^{-1}$ ). 

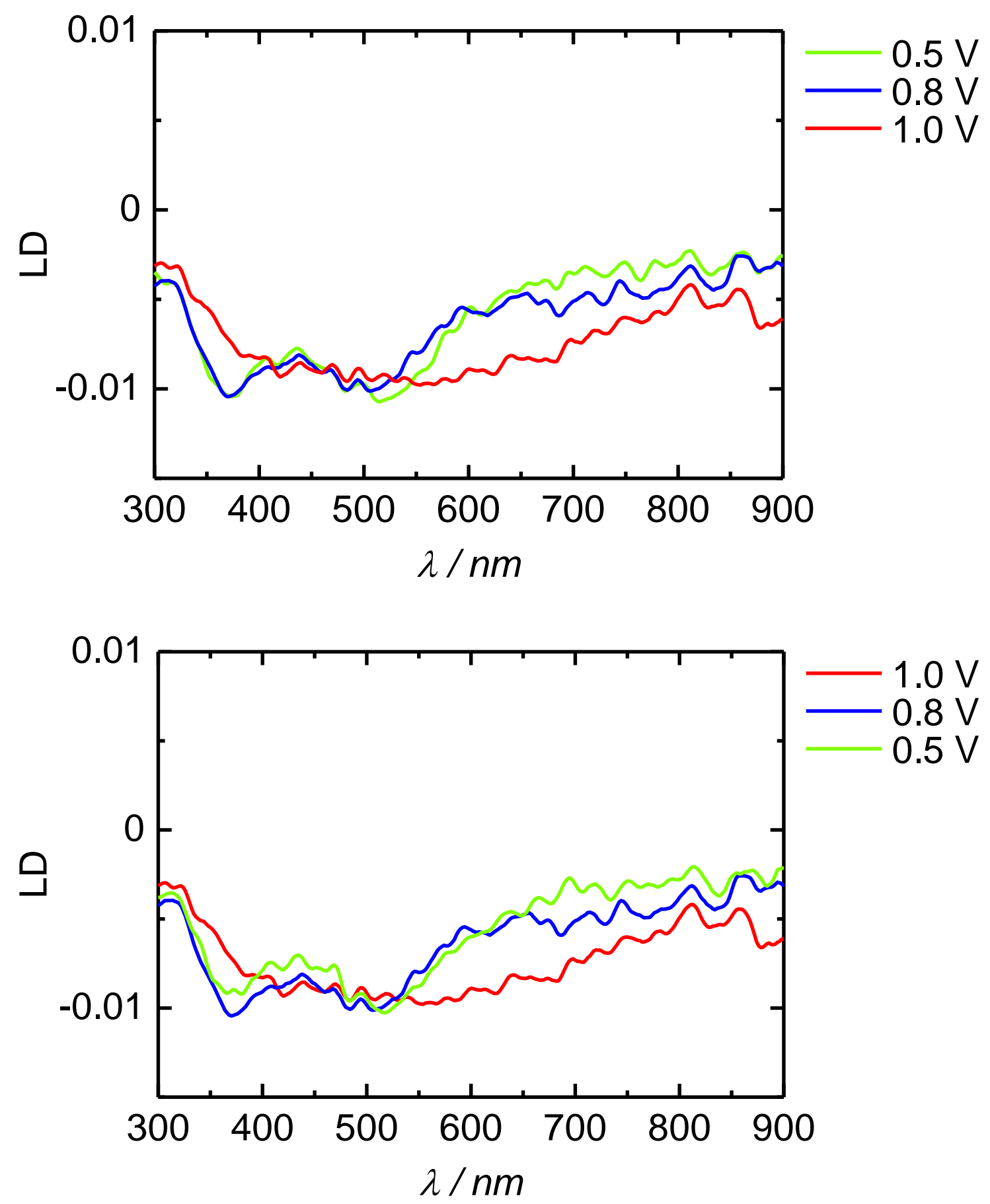

Fig. 8. LD spectra of (R)-PTBT 4 T at various applied potentials vs. $\mathrm{Ag} / \mathrm{Ag}^{+}$reference electrode during oxidation process (upper) and reduction process (lower). 